\title{
Particle yields from numerical simulations
}

\author{
Marietta M. Homor ${ }^{*}$ and Antal Jakovác ${ }^{\dagger}$ \\ Institute of Physics, Eötvös Loránd University, 1/A Pázmány P. Sétány, H-1117 Budapest, Hungary
}

(Received 18 October 2017; published 10 April 2018)

\begin{abstract}
In this paper we use numerical field theoretical simulations to calculate particle yields. We demonstrate that in the model of local particle creation the deviation from the pure exponential distribution is natural even in equilibrium, and an approximate Tsallis-Pareto-like distribution function can be well fitted to the calculated yields, in accordance with the experimental observations. We present numerical simulations in the classical $\Phi^{4}$ model as well as in the SU(3) quantum Yang-Mills theory to clarify this issue.
\end{abstract}

DOI: 10.1103/PhysRevD.97.074504

\section{INTRODUCTION}

In collider experiments the observed hadron yields are surprisingly far from the Boltzmann distribution expected from blackbody radiation of the hot plasma. It is true for all yields coming from hadron collisions, starting from $\mathrm{p}-\mathrm{p}$ collisions in ALICE, CMS, STAR and PHENIX collaborations, respectively [1-4]. Part of these particle spectra can be explained by perturbative QCD calculations [5], but for a complete description of all of the fits a Tsallis-Pareto-like [6] Ansatz is necessary. These fits have just one additional parameter compared to a Boltzmannian, yet they work very nicely in experimental fits [2,7-9] although in certain cases the soft and hard physics has to be treated separately [10].

There are several interpretations of these results. A QCDbased generalized Ansatz [11-13] results in distribution functions that are similar to Tsallis distribution. A more natural explanation is to assume some collective behavior that leads to these types of distributions. The reason for the deviation from the Boltzmann distribution can be the finite volume [14], fluctuating temperature $[15,16]$. In fact the leading deviation from Boltzmann distribution is of Tsallis form [17]. It is also possible that the event-by-event distributions are Boltzmannian, but the hadron multiplicities fluctuate according to negative binomial distribution, and only in the cumulative yields do we see Tsallis distribution [10].

Our main motivation in this paper is to support the experimental evidences found by [1-4] by numerical simulations, and to provide an alternative mechanism to

\footnotetext{
homor.marietta.m@gmail.com

jakovac@caesar.elte.hu
}

Published by the American Physical Society under the terms of the Creative Commons Attribution 4.0 International license. Further distribution of this work must maintain attribution to the author(s) and the published article's title, journal citation, and DOI. Funded by SCOAP ${ }^{3}$. produce Tsallis distribution. Usually it is believed that if the microscopic distribution is Boltzmannian (i.e. we have a canonical ensemble), then also the particle yields are Boltzmann distributed. This is, however, not true-the distribution of the particle yields depends on the particle creation mechanism.

To understand this statement we recall that in weakly interacting gases, like the photon gas that interacts only with the wall of the cavity, the particle states are (almost) the same as the energy eigenstates: they have definite momentum and energy, and they extend to the whole cavity. If a photon escapes from the system it carries information about the distribution of the energy eigenstates, and so, correspondingly, we obtain a photon yield that is distributed according to the Bose-Einstein distribution.

In strongly interacting plasmas, however, the situation changes. Particle states are no longer energy eigenstates, in fact they consist of a lot of energy eigenstates with a Lorentzian envelope: they are quasiparticles. Moreover, they are basically local objects, they do not extend to the whole plasma. The phenomenon of jet quenching [18] clearly indicates that high energy particles are created locally, in a volume of at most of order $1 \mathrm{fm}$. As a consequence, if they escape the plasma, they do not carry information about the occupation of the global energy levels, but about the local energy density. The fluctuation of the energy in a small volume, however, is different than in a large subsystem, it need not (and actually does not) follow Boltzmann distribution.

According to this picture to assess the particle yields coming from a strongly interacting plasma we have to measure the distribution of the local energy density. This is a measurable quantity even in numerical simulations, making possible to give predictions on the observable yields which are otherwise very hardly accessible quantities.

In this paper we have considered two models basically to demonstrate the method: a classical $\Phi^{4}$ model and a quantum SU(3) Yang-Mills gauge theory. As it turns out, in both cases 
the distribution of the local energy density stabilizes relatively fast, well before the actual thermal equilibration, and a Tsallis distribution is an excellent fit to them. In the process of approaching equilibrium, the parameters of the Tsallis distribution change. In the classical scalar model case it is possible to follow real time evolution, and so the variation of the temperature and Tsallis parameter in real time. In the following we discuss the histogram method to determine the local energy distribution function, consider the classical quartic model and the quantum Yang-Mills model, and finally we close the paper with a Conclusion section.

\section{LOCAL ENERGY DENSITY DISTRIBUTION}

As stated in the Introduction, our aim is to determine the local energy density distribution. In this section, we recall some basic definitions of probability theory.

First of all, let $X$ be a stochastic variable. The indicator of $X$ being in the $[x, x+\Delta x]$ interval is $\square_{[x, x+\Delta x]}(X)=\Theta(X-x) \Theta(x+\Delta x-X)$, where $\Theta$ is the Heaviside function. The expectation value of this indicator equals the probability of $X$ being in the interval:

$$
\left\langle\square_{[x, x+\Delta x]}(X)\right\rangle=\mathcal{P}(X \in[x, x+\Delta x]) .
$$

In the statistical approach we take the expectation value above some configuration space, then we assume that $X$ is a function(al) of the configurations $X(A)$. If the distribution of the configurations $A$ is given, let us denote it by $f(A)$, then the expectation value of a general quantity $R(A)$ can be computed as

$$
\langle R\rangle=\int \mathcal{D} A R(A) f(A)
$$

In canonical ensemble $f(A)=\exp (-\beta H(A)) / Z$. In practice, however, we generate a lot of configurations according to the distribution function $f(A)$, either by solving the equation of motion (classical theory), by following a Markov-process (Monte Carlo simulations) or considering small subsystems of a configuration in a large volume. In any case we have $A_{1}, A_{2}, \ldots, A_{n}$ configurations and we take the expectation value by summing above them:

$$
\langle R\rangle=\frac{1}{n} \sum_{i=1}^{n} R\left(A_{i}\right) .
$$

Therefore the expectation value of the indicator is proportional to the number of configurations $n_{i}$ where the $X\left(A_{i}\right)$ quantity has values between $x$ and $x+\Delta x$. Therefore

$$
\mathcal{P}(X \in[x, x+\Delta x])=\frac{n_{i}}{n} .
$$

To determine the complete distribution function, therefore, we divide the possible outputs of $X$ to bins, each of them is
$\Delta x$ wide. Then we scan over all available configurations, and each configuration contributes to the bin that contains $X\left(A_{i}\right)$. This provides a histogram that is exactly the desired $\mathcal{P}(X \in[x, x+\Delta x])$ distribution function.

The limit $\Delta x \rightarrow 0$ provides the probability density of the variable $X$ :

$$
p(x)=\lim _{\Delta x \rightarrow 0+} \frac{\mathcal{P}(X \in[x, x+\Delta x])}{\Delta x} .
$$

If we write (1) into the definition (5) of $f(x)$, then we get a Dirac- $\delta$ approximation and we can write rather formally:

$$
p(x)=\langle\delta(X-x)\rangle .
$$

In this work, the quantity in question-in other words the stochastic variable-is the local energy density $\epsilon_{x}$ where $x$ is an arbitrary space-time coordinate. With this (6) becomes

$$
p(\epsilon)=\left\langle\delta\left(\epsilon_{x}-\epsilon\right)\right\rangle .
$$

It is important to note that the energy distribution of a small subsystem of a thermal system need not follow Boltzmann distribution. The reason is that the total energy of the system can be written as $E=E_{\text {subsystem }}+E_{\text {environment }}+\delta E$, where $\delta E$ "surface energy" depends both on the state of the subsystem and the environment. If the volume of the subsystem is not large enough, then $\delta E$ cannot be neglected, this leads to non-Boltzmannian energy distribution. Only in largish volumes which are small compared to the complete system, but large enough to neglect the surface energy terms, can we deduce that the probability density of measuring a given energy value is Boltzmann distributed.

\section{A. Local energy distribution in free systems}

It is worth thinking about the form of the local energy distribution when it is Boltzmann-like. So let us assume that $p(\epsilon)=\mathcal{N} e^{-c \epsilon}$ with some constants. We have two constraints:

$$
1=\int_{0}^{\infty} d \epsilon p(\epsilon), \quad\left\langle\epsilon_{x}\right\rangle=\int_{0}^{\infty} \operatorname{d\epsilon \epsilon } p(\epsilon),
$$

these fix the constants to be

$$
p(\epsilon)=\frac{1}{\left\langle\epsilon_{x}\right\rangle} e^{-\epsilon /\left\langle\epsilon_{x}\right\rangle} .
$$

Therefore we do not expect $e^{-\beta \epsilon}$ form, only if $\left\langle\epsilon_{x}\right\rangle=T$.

In simple systems we in fact obtain such a form. Most simply, in a system built up from local independent systems we have for all configurations $\sigma$ :

$$
E(\sigma)=\sum_{i} \epsilon_{i}\left(\sigma_{i}\right) .
$$


In this case, using $Z_{i}=\sum_{\sigma_{i}} e^{-\beta \epsilon_{i}}$ we find

$$
p(\epsilon)=\frac{1}{Z} \sum_{\sigma} e^{-\beta E} \delta\left(\epsilon-\epsilon_{i}\right)=\frac{1}{Z_{i}} \sum_{\sigma_{i}} e^{-\beta \epsilon_{i}} \delta\left(\epsilon-\epsilon_{i}\right)=\frac{1}{Z} e^{-\beta \epsilon},
$$

because at sites $j \neq i$ the corresponding $Z_{j}$ factors drop out.

We also have the same results in case of free systems, even when the energy is the sum of the momentum states, while the local energy density is localized in real space. To prove this statement we first rewrite the energy density distribution as

$p(\epsilon)=\Theta(\epsilon) \int_{-\infty}^{\infty} d \lambda\left\langle e^{i \lambda\left(\epsilon-\epsilon_{x}\right)}\right\rangle=\Theta(\epsilon) \int_{-\infty}^{\infty} d \lambda e^{i \lambda \epsilon}\left\langle e^{-i \lambda \epsilon_{x}}\right\rangle$,

where we assumed $\epsilon_{x}>0$ for all configurations. Then we expand the exponential

$$
\left\langle e^{-i \lambda \epsilon_{x}}\right\rangle=\sum_{\ell=0}^{\infty} \frac{(-i \lambda)^{\ell}}{\ell !}\left\langle\epsilon_{x}^{\ell}\right\rangle .
$$

To avoid UV divergences we renormalize the above expression taking the normal ordered product, so we calculate

$$
\left\langle: \epsilon_{x}^{\ell}:\right\rangle
$$

The local energy density can be defined in a number of ways, each definition differs from each other in total divergences. We will choose a simple representation, where the local energy density can be written as a function of the creation-annihilation operators $a_{p}$ and $a_{p}^{\dagger}$ in $d$ dimensions as

$$
: \epsilon_{x}:=\int \frac{d^{d} p}{(2 \pi)^{d}} \frac{d^{d} q}{(2 \pi)^{d}} \sqrt{\omega_{p} \omega_{q}} e^{i(p-q) x} a_{q}^{\dagger} a_{p} .
$$

It is simple to see that

$$
: H:=\int d^{d} x: \epsilon_{x}:=\int \frac{d^{d} p}{(2 \pi)^{d}} \omega_{p} a_{p}^{\dagger} a_{p} .
$$

Now we can compute the expectation value in question at $x=0$ :

$$
\begin{aligned}
\left\langle: \epsilon_{x=0}^{\ell}:\right\rangle= & \frac{1}{Z} \sum_{\{n\}} e^{-\beta E_{n}} \int \prod_{i=1}^{\ell} \frac{d^{d} p_{i}}{(2 \pi)^{d}} \frac{d^{d} q_{i}}{(2 \pi)^{d}} \sqrt{\omega_{p_{i}} \omega_{q_{i}}} \\
& \times\left\langle n\left|a_{q_{1}}^{\dagger} \cdots a_{q_{\ell}}^{\dagger} a_{p_{1}} \cdots a_{p_{\ell}}\right| n\right\rangle,
\end{aligned}
$$

where $E_{n}=\langle n|: H:| n\rangle$. Since the same state stands in the left- and right-hand side of the expectation value, we must have the same number of creation and annihilation operators for each momenta. We will omit the possibility that more than two operators have the same momenta, since these contributions are suppressed by factors of $V_{d}$ the volume of the $d$ dimensional space. This means that we have to make pairs (Wick theorem). It is easy to see that all pairings give the same contribution, so finally we have

$$
\left\langle n\left|a_{q_{1}}^{\dagger} \cdots a_{q_{\ell}}^{\dagger} a_{p_{1}} \cdots a_{p_{\ell}}\right| n\right\rangle=\ell ! \prod_{i=1}^{\ell} n_{p_{i}}(2 \pi)^{d} \delta\left(p_{i}-q_{i}\right) .
$$

Substituting back this result we see that the expectation value of the $\ell$-times local energy density is proportional to the expectation value of the local energy density to the $\ell$ th power:

$$
\left\langle: \epsilon_{x=0}^{\ell}:\right\rangle=\ell !\left\langle: \epsilon_{x=0}:\right\rangle^{\ell}
$$

Therefore

$$
\left\langle e^{-i \lambda \epsilon_{x}}\right\rangle=\sum_{\ell=0}^{\infty}(-i \lambda)^{\ell}\left\langle\epsilon_{x}\right\rangle^{\ell}=\frac{1}{1+i \lambda\left\langle\epsilon_{x}\right\rangle},
$$

and so the inverse Fourier transform yields

$$
p(\epsilon)=\frac{1}{\left\langle\epsilon_{x}\right\rangle} e^{-\epsilon /\left\langle\epsilon_{x}\right\rangle},
$$

which means that in the free systems the local energy density is indeed Boltzmann distributed.

We see from this calculation that the validity of the Boltzmann distribution depends on very sensitive details, for example that the expectation value of powers of the local energy density is proportional to powers of the expectation value of the local energy density [cf. Eq. (19)]. In a general theory it will not be true anymore, resulting that $\left\langle e^{-i \lambda \epsilon_{x}}\right\rangle$ is not a simple pole and then $p(\epsilon)$ is no longer exponential. We cannot determine the actual form, but based on very general arguments [17] we expect that if the deviation is small, then it must be a Tsallis-Pareto distribution.

In the following sections, we consider the real time simulation of the classical $\Phi^{4}$ theory in three dimensions and perform a standard Monte Carlo simulation with heatbath algorithm for the Euclidean SU(3) gauge theory.

\section{A TOY MODEL: CLASSICAL $\Phi^{4}$ THEORY}

Our first toy model is the well-known classical $\Phi^{4}$ theory. One of the advantages of classical theories is that we can perform real-time simulations by successively solving the canonical equations and we can calculate physical quantities that are hardly accessible in other methods. Classical theories are used to approach the full theory in a lot of contexts [19-28]. 
The discretized version has the Hamiltonian [28]

$$
H=\sum_{x \in U} \epsilon_{x},
$$

where $U$ denotes the discretization mesh (in our case a cubic lattice with $N$ sites in all directions, $N=40,50$ ), and $\epsilon_{x}$ is the local energy density

$$
\epsilon_{\mathbf{x}}=\frac{1}{2} \Pi_{\mathbf{x}}^{2}+\frac{1}{2}(\nabla \Phi)_{\mathbf{x}}^{2}+\frac{m^{2}}{2} \Phi_{\mathbf{x}}^{2}+\frac{\lambda}{24} \Phi_{\mathbf{x}}^{4} .
$$

In this expression we have to use the discretized gradient $\nabla_{i} \Phi(x)=a^{-1}\left[\Phi\left(x+a e_{i}\right)-\Phi(x)\right]$, where $a$ is the discretization spacing, and $e_{i}$ is the unit vector pointing to the $i$ th direction. The corresponding equations of motion read

$$
\dot{\Phi}=\Pi, \quad \dot{\Pi}=\triangle \Phi-m^{2} \Phi-\frac{\lambda}{6} \Phi^{3},
$$

where $\triangle \Phi=a^{-2} \sum_{i=1}^{3}\left[\Phi\left(x+a e_{i}\right)+\Phi\left(x-a e_{i}\right)-2 \Phi(x)\right]$ is the discretized Laplacian. The continuous equation of motion preserves energy, but in the time discretized version the energy conservation depends on the algorithm. We used leapfrog and Runge-Kutta methods; for further discussion cf. [28].

We note that the system can be rescaled as $t \rightarrow t / a$, $\Phi \rightarrow \sqrt{\lambda} a \Phi, \Pi \rightarrow \sqrt{\lambda} a^{2} \Pi$, then we have the same equations of motion with $a=1, m \rightarrow a m$ and $\lambda=1$. This means that the value of $\lambda$ does not modify the classical dynamics, it can be compensated by the normalization of the fields. The energy density rescales as $\epsilon \rightarrow \lambda a^{4} \epsilon$. In the simulations we have used the $a=1$ unit, but we have kept the value of $\lambda$ to test the numerical effects.

After thermalization we can use the thermodynamical notions. The temperature $(T)$ of the system is defined as [28]

$$
T=\frac{1}{2 N^{3}}\left\langle\left|\Pi_{k}\right|^{2}\right\rangle,
$$

where $N^{3}$ is the number of lattice sites and $\Pi_{k}$ is the Fouriertransformed momentum field. We use this formula to check whether the system reached thermal equilibrium by verifying that $\left\langle\left|\Pi_{k}\right|^{2}\right\rangle$ is independent of $\mathbf{k}$ (equipartition). It turned out that we can distinguish two timescales, as higher modes thermalize much faster than low ones. After 10000 time steps, the system can be considered fully thermalized.

\section{A. Numerical results for the classical $\Phi^{4}$ theory}

We have solved the classical EoM on $40^{3}$ and $50^{3}$ lattices. These are the largest volumes we had, the thermodynamic limit is already reached at these volumes (cf. [28]). As initial conditions we have chosen $\Phi_{x}=0$ for every point, and we have assumed pointwise independent distributions for the canonical momenta. In one case the momentum distribution was a uniform distribution in the $[0,1]$ range, in the other case we had a $1 / \cosh (x)$ distribution. In the simulation we have chosen $d t=0.1$ time step in lattice spacing units. We checked that the results are not sensitive to other choices of the time step as well as choices of the simulation algorithm. We have chosen $m^{2}=-0.5$ for the mass parameter, but due to the renormalization properties of the classical $\Phi^{4}$ theory different choices for the "bare" mass correspond to the same system at different temperatures [28].

After starting the simulation, the distribution of the local energy density very quickly stabilizes. Already after the 17th time step the histograms reach the characteristic form which remained true in all later times. We can see these distributions in Figs. 1 and 2. Each data point in the histogram is the average of 50 runs with the same initial

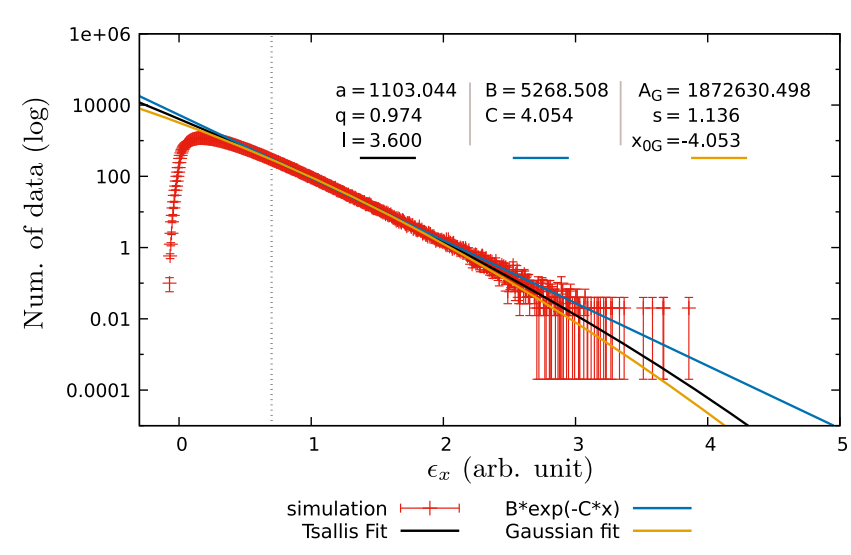

FIG. 1. Local energy-density histogram with Boltzmann (blue line), Tsallis (black line) and Gaussian (yellow) fits on semilog-scale after 17 time steps. The simulation was started with a random initial condition for $\Pi_{\mathbf{x}}$. Data points are averaged from 50 simulations and shown with their standard error.

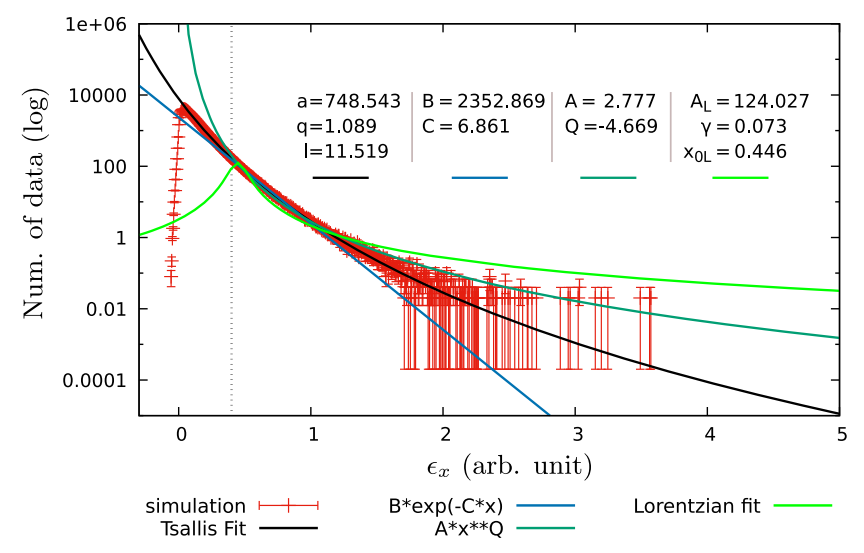

FIG. 2. Local energy-density histogram with simple power function (green line), Boltzmann (blue line), Lorentzian (green) and Tsallis (black line) fits on a semi-log-scale after 17 time steps. The simulation was started with a secant hyperbolic initial condition for $\Pi_{\mathbf{x}}$. Data points are averaged from 50 simulations and shown with their standard error. 
condition and the error bars represent the standard error of the mean (SEM). We tried several fit functions to describe the functional form of the distribution (exponential i.e. Boltzmannian, pure power law, Gaussian, Lorentzian) which for certain energy ranges and for certain cases in fact can reproduce the data. But by our experience as an overall fewparameter fit the Tsallis-Pareto distribution is singled out:

$$
\mathcal{P}(\varepsilon)=a[1+(q-1) \beta \varepsilon]^{\frac{1}{1-q}} .
$$

Note that for $q \rightarrow 1$ it gives back the Boltzmann distribution. The actual values, $q=0.974$ and $q=1.094$ respectively, are very close to the Boltzmannian case, and so it can be revealed only by a thorough analysis with at least $10^{6}$ independent data points.

In the 17th time step the system is very far from equilibrium, but the Tsallis-Pareto distribution of the energy density remained true, with time dependent Tsallis parameter $q(t)$. This function is plotted in Figs. 3 and 4 for two different time intervals, starting with different total energy

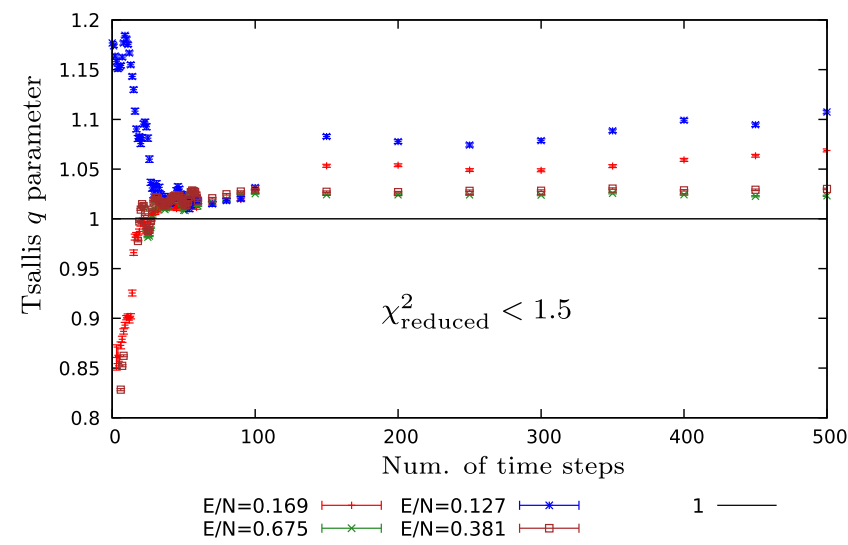

FIG. 3. Time dependence of the Tsallis parameter at four different total energies with various initial conditions. The first 500 time steps.

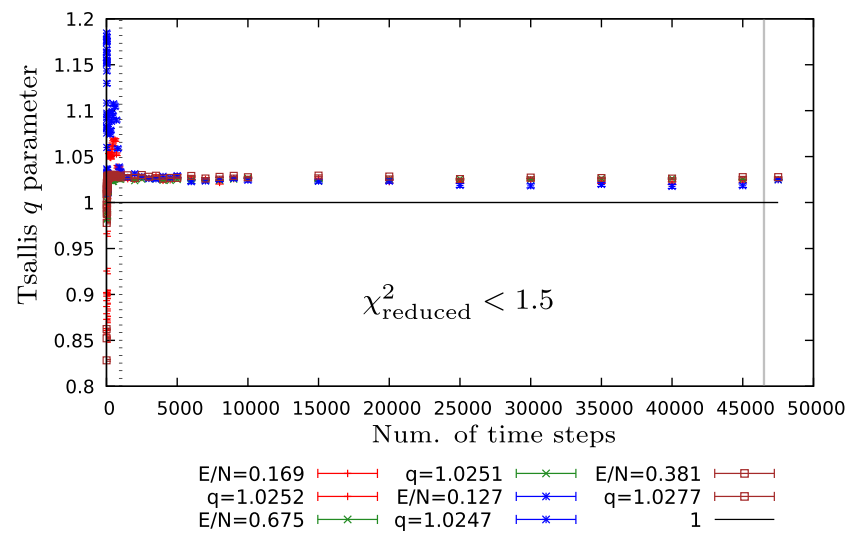

FIG. 4. Time dependence of the Tsallis parameter at four different total energies with various initial conditions. The last points indicate the average values for each energy from 1000 to 45000 time steps. (corresponding to different temperatures after thermalization and different lattice sizes). In the plots we only presented those points for which the Tsallis fit had $\chi^{2} /$ dof $<1.5$. We can observe that even in those cases when $q$ started from a value smaller than 1 , finally in all runs it reached a value that is consistently larger than 1 . It is interesting that this value seems to be independent on the temperature as well as on the lattice sizes we studied. The Tsallis parameter takes its equilibrium value already in the prethermalized state (i.e. when only higher modes are thermalized) within error. The actual value of the equilibrium Tsallis parameter is obtained by averaging the $q$ values after time step 1000, assuming there is a single parameter for all runs. We obtain $q=$ $1.026 \pm 0.0012$ which is in the order of the experimental values.

At this stage it is not clear, whether the deviation from the Boltzmann distribution is a property of the energy density only, or the microscopic distribution function $f(A)$ over the configurations [cf. (2)] is also non-Boltzmannian. To this end we have carefully studied the distribution of $\Pi_{x}^{2}$ for different energy values and different lattice sizes with large statistics. If the thermal ensemble has a distribution function $e^{-\beta H}$, then the $x=\Pi_{x}^{2} / 2$ values for a given $x$ must follow $e^{-\beta x}$ exponential distribution, i.e. we must have $q=1$ in the Tsallis fit. The result for the averaged data is $q_{\text {momentum }}=$ $0.999 \pm 0.001$ for all the cases we studied. This means that the distribution over the configurations is in fact the standard Boltzmann distribution, and only the energy density has a Tsallis-like distribution. Learning this fact we could proceed to the quantum field theory case, where only the equilibrium can be studied with Monte Carlo methods, but the distribution of the energy density is expected to be Tsallis-like even in this case.

\section{EUCLIDEAN SU(3) PURE GAUGE THEORY}

Our second model is the quantum SU(3) Yang-Mills theory. The action of the model in Euclidean formalism is the following:

$$
S_{\mathrm{YM}}=\frac{1}{4} \int d^{4} x F_{\mu \nu}^{a} F_{\mu \nu}^{a},
$$

where $F_{\mu \nu}(x)=-\mathfrak{i} g F_{\mu \nu}^{a}(x) T_{a}$ is the gluon field strength tensor, $T_{a}$ are the generators of the Lie algebra and $g$ is the coupling constant.

We use the Wilson action for the lattice formulation of the theory:

$$
S[U]=\sum_{p} \beta\left(1-\frac{1}{N} \operatorname{Re} \operatorname{Tr} U_{p}\right),
$$

where $U(x+\mu, x)$ is the parallel transporter from lattice coordinate $x$ to $x+\mu$ and the plaquette variable corresponding to $x$ is the product of four parallel transporters along the closed path $x \rightarrow x+\mu \rightarrow x+\mu+\nu \rightarrow x+\nu \rightarrow x$. 
It is well known that the Wilson action corresponds to the continuum theory if one chooses $\beta=2 N / g^{2}$ (in our case $N=3$ ) and the connection between the parallel transporters and the gauge fields is $U(x, \mu)=\mathrm{e}^{-a A_{\mu}(x)}$. The continuum limit is reached as $\beta \rightarrow \infty$.

The local energy density is now given by the plaquette energy:

$$
\epsilon=\left\langle 1-\frac{1}{\operatorname{Tr} \rrbracket} \operatorname{Tr} U_{p}\right\rangle
$$

We determine the histogram of the local energy density in the same way as we have done in the classical theory, picking out independent configurations from the thermal ensemble.

An advantage of the histogram method is that renormalization can be explicitly traced in the distributions. In the case of the local energy density, being a composite operator, we expect a multiplicative renormalization as well as an eventual mixing with the unit operator (additive renormalization). In the histograms the two types of renormalization show up as a dilatation and a position shift. Neither of these effects modify the power of the high-energy tail: therefore the Tsallis parameter is not renormalized.

We use Monte Carlo simulation with the well-known heat-bath algorithm to determine the distribution of $\epsilon$ with zero energy initial condition (i.e. $\epsilon_{p}=0$ for all plaquettes) at lattices $N_{t} \times N_{s}^{3}$.

For the fits of the resulting distributions we have taken into account the reduction of the phase space at low energy densities. As a result, we modify our previous fit function (26) by a factor of $x^{n}$ where $n \approx 3$ :

$$
f(x)=a x^{n}(1+(q-1) l x)^{\frac{1}{1-q}} .
$$

The error of the histogram is assumed to be a Gaussian (meaning $\sqrt{N}$ standard deviation for a bin which contains $N$ points). However, in more realistic models it would be advised to perform a maximum likelihood parameter estimation (in the context of Tsallis distributions cf. [29]).

\section{A. Plaquette energy histogram}

A typical plaquette energy histogram is presented in Fig. 5. In the figure the best Tsallis (30) and Boltzmannian $g(x)=A x^{N} \mathrm{e}^{-B x}$ fits can also be seen. It is evident that the Tsallis fit is better than the Boltzmannian one, similarly as in the case of classical $\Phi^{4}$ theory.

\section{B. Tsallis $q$ for various $\beta$}

The evolution of the Tsallis parameter (with its fit error) is presented in Fig. 6 as a function of the Monte Carlo time. We covered a wide range of $\beta$, starting from $\beta=6$ to values as high as $\beta=20$. Note that the plaquette energy has an upper bound [due to the properties of the trace of $\mathrm{SU}(3)$

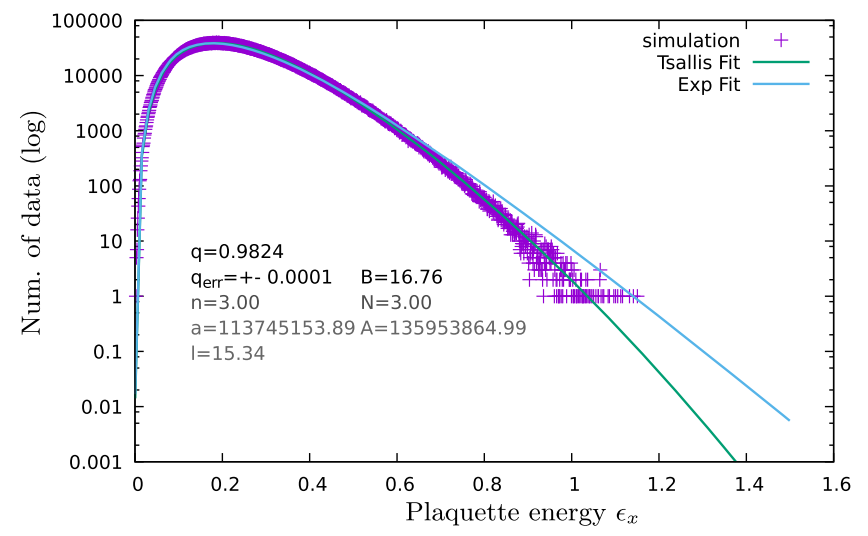

FIG. 5. Plaquette energy histogram on semi-log-scale after 15 heat-bath sweeps at $\beta=8, N_{t}=8, N_{s}=60$. The blue line is the Boltzmann and the green line is the Tsallis fit.

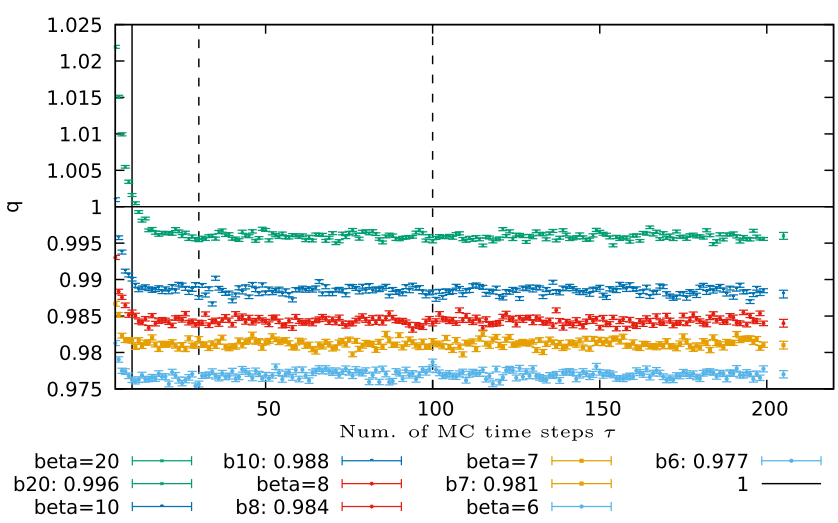

FIG. 6. Tsallis $q$ parameter during MC sweeps for various $\beta$, with $N_{t}=2$ and $N_{s}=50$. The last, slightly separated points show the average from $\tau=100$ with their statistical error. The average values are present in the legend.

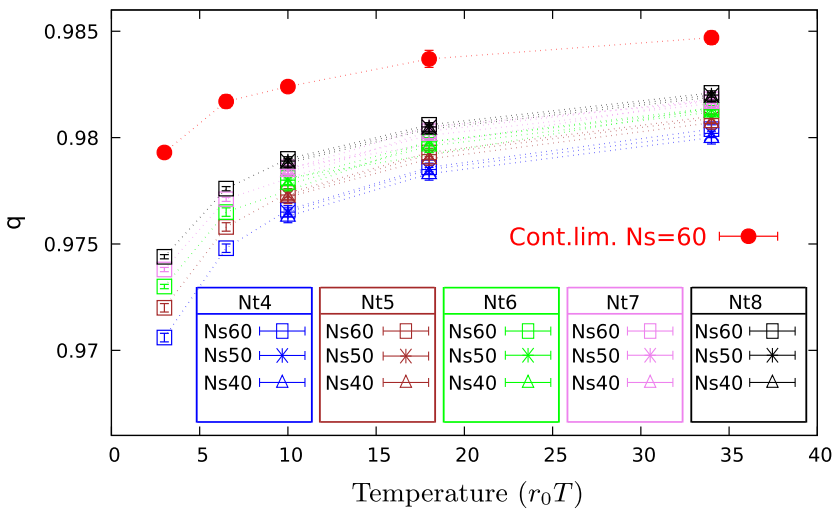

FIG. 7. Temperature dependence of $q$ for various $N_{t}$ and $N_{s}$. Dashed lines are to guide the eye.

matrices], and this severely distorts the histogram below $\beta=6$.

It is interesting that the shape of the distribution shows up very early, well before thermalization: already after the 


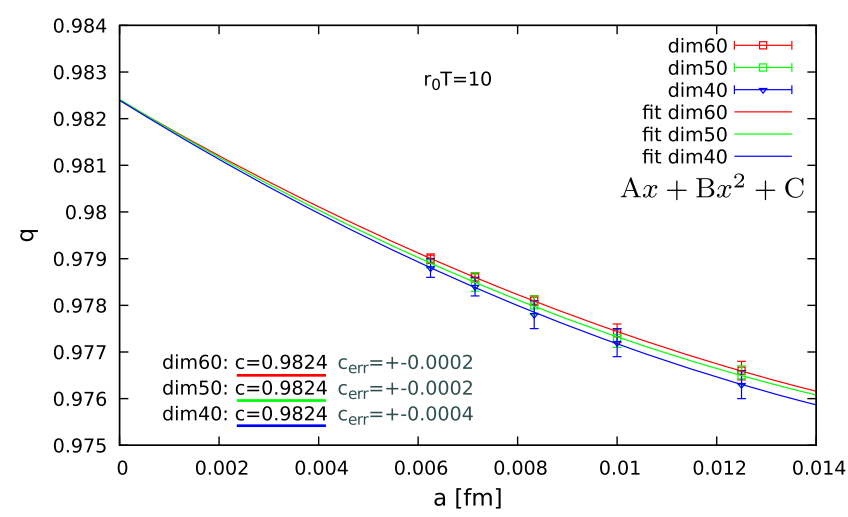

FIG. 8. Tsallis $q$ parameter vs lattice spacing at $r_{0} T=10$ fixed temperature. A second order polynomial fit is performed to the numeric data. Three different lattice sizes are taken into consideration. Red: $N_{s}=60$; green: $N_{s}=50$; and blue: $N_{s}=40$.

first MC sweep we find a roughly Tsallis-like distribution, although the Tsallis fit converges well after about $\tau=10$ (first vertical line in Fig. 6). Thermalization time is $\tau \approx 30$ (second vertical line in Fig. 6). We have calculated the equilibrium Tsallis parameter from configurations after $\tau=100$ (third vertical line) for each simulation.

We see that the Tsallis parameter $q \rightarrow 1$ as $\beta \rightarrow \infty$. But this also means that the lattice spacing $a \rightarrow 0$, and so also the physical volume goes to zero. That means that we cannot reach the thermodynamic limit with the available lattice volumes.

\section{Continuum limit}

To connect the numerical observations to physical units, we have to fix the scale. We have chosen the Sommer scale [30] and interpolated the $\beta$ and lattice constant $a$ relation based on the data from [31-33]. The dimensionless temperature is as follows: $r_{0} T=\frac{1}{N_{t}} \frac{r_{0}}{a}$, where $r_{0} \approx 0.5 \mathrm{fm}$ is the Sommer-scale parameter.

The temperature dependence of the $q$ parameter is shown in Fig. 7. Five different $N_{t}$ values are considered. To check the thermodynamic limit, we repeated the simulation for $N_{s}=60, N_{s}=50$ and $N_{s}=40$.

In this figure also the continuum limit is shown. We approached the continuum limit in a way that at fixed temperature and at fixed $N_{s}$ we plotted the $q$ results for different lattice spacings. Then we used a second order polynomial to fit the numeric data and determined the $a \rightarrow 0$ limit. For a demonstrative example we present the result for $r_{0} T=10$ in Fig. 8. Interestingly enough, we got $q<1$ as opposed to the case of the classical $\Phi^{4}$ theory, though the absolute difference from 1 is approximately the same.

\section{CONCLUSIONS AND OUTLOOK}

The particles emerging from a strongly interacting plasma are created locally and so they carry information about the local energy density. The distribution of this quantity is in general different from the canonical energy level distribution, except for the free (or very weakly interacting) theories. Therefore in the particle yields coming from a strongly interacting plasma we should not expect Boltzmann distribution. For the actual expectation we have to measure the distribution of the local energy density.

In this work we have determined the local energy density distribution with the histogram method in the classical $\Phi^{4}$ and in the quantum SU(3) Yang-Mills theory. In both cases the energy level distribution is Boltzmannian, but we have found that the Boltzmann distribution does not fit well to the local energy distribution in either case. However, the Tsallis distribution is a good fit, similarly to experimental data. The corresponding Tsallis parameter differs significantly from 1 . Thermodynamic limit analysis is performed in both cases and we carried out the continuum limit analysis as well for SU(3) gauge theory. We remark that the renormalization of the local energy density does not affect the power of the power law tail, i.e. the Tsallis parameter $q$ receives no renormalization correction.

We have found that in the case of the classical $\Phi^{4}$ theory, the Tsallis parameter $q=1.024 \pm 0.001$-this is in the order of the experimental values obtained from heavy ion collisions. Regarding the $\mathrm{SU}(3)$ gauge theory, $q=0.9835 \pm$ 0.0005 . Interestingly it is smaller than 1 , although $|1-q|$ is in the same order of magnitude in both models.

These results encourage us to proceed to our main goal, namely to perform similar analysis for QCD and to compare the results with experimental data.

\section{ACKNOWLEDGMENTS}

The authors acknowledge useful conversations with A. Patkos, T. S. Biro and C. Tsallis. This work was supported by the Hungarian Research Fund (OTKA) under Contracts No. K104292 and No. K123815. 
[1] K. Aamodt et al. (ALICE Collaboration), Production of pions, kaons and protons in $p p$ collisions at $\sqrt{s}=900 \mathrm{GeV}$ with ALICE at the LHC, Eur. Phys. J. C 71, 1655 (2011).

[2] V. Khachatryan et al. (CMS Collaboration), TransverseMomentum and Pseudorapidity Distributions of Charged Hadrons in $p p$ Collisions at $\sqrt{s}=7 \mathrm{TeV}$, Phys. Rev. Lett. 105, 022002 (2010).

[3] B. I. Abelev et al. (STAR Collaboration), Strange particle production in $p+p$ collisions at $\sqrt{s}=200 \mathrm{GeV}$, Phys. Rev. C 75, 064901 (2007).

[4] A. Adare et al. (PHENIX Collaboration), Identified charged hadron production in $p+p$ collisions at $\sqrt{s}=200$ and 62.4 GeV, Phys. Rev. C 83, 064903 (2011).

[5] G. G. Barnaföldi, J. Barrette, M. Gyulassy, P. Levai, and V. Topor Pop, Predictions for $p+\mathrm{Pb}$ at $4.4 \mathrm{ATeV}$ to test initialstate nuclear shadowing at energies available at the CERN large hadron collider, Phys. Rev. C 85, 024903 (2012).

[6] C. Tsallis, Possible generalization of Boltzmann-Gibbs statistics, J. Stat. Phys. 52, 479 (1988).

[7] V. Khachatryan et al. (CMS Collaboration), Transverse momentum and pseudorapidity distributions of charged hadrons in pp collisions at $\sqrt{s}=0.9$ and $2.36 \mathrm{TeV}$, J. High Energy Phys. 02 (2010) 041.

[8] G. G. Barnafoldi, K. Urmossy, and T. S. Biro, TsallisPareto-like distributions in hadron-hadron collisions, J. Phys. Conf. Ser. 270, 012008 (2011).

[9] J. Cleymans and D. Worku, The Tsallis distribution in proton-proton collisions at $\sqrt{s}=0.9 \mathrm{TeV}$ at the LHC, J. Phys. G 39, 025006 (2012).

[10] K. Urmossy, G. G. Barnaföldi, S. Harangozó, T. S. Biró, and Z. Xu, A 'soft + hard' model for heavy-ion collisions, J. Phys. Conf. Ser. 805, 012010 (2017).

[11] R. Hagedorn, Multiplicities, $p_{T}$ distributions and the expected hadron $\rightarrow$ quark-gluon phase transition, Riv. Nuovo Cimento 6, 1 (1983).

[12] C. Y. Wong, G. Wilk, L. J. L. Cirto, and C. Tsallis, Possible implication of a single nonextensive $p_{T}$ distribution for hadron production in high-energy $p p$ collisions, EPJ Web Conf. 90, 04002 (2015).

[13] C. Y. Wong, G. Wilk, L. J. L. Cirto, and C. Tsallis, From QCD-based hard-scattering to nonextensive statistical mechanical descriptions of transverse momentum spectra in high-energy $p p$ and $p \bar{p}$ collisions, Phys. Rev. D 91, 114027 (2015).

[14] T. S. Biro, Ideal gas provides q-entropy, Physica (Amsterdam) 392A, 3132 (2013).

[15] G. Wilk and Z. Wlodarczyk, Consequences of temperature fluctuations in observables measured in high energy collisions, Eur. Phys. J. A 48, 161 (2012).

[16] T. S. Biro, P. Van, G. G. Barnafoldi, and K. Urmossy, Statistical power law due to reservoir fluctuations and the universal thermostat independence principle, Entropy 16, 6497 (2014).
[17] T. S. Biro, G. Purcsel, G. Gyorgyi, and A. Jakovac, A nonconventional description of quark matter, J. Phys. G 31, S759 (2005).

[18] U. A. Wiedemann, Jet quenching in heavy ion collisions, edited by R. Stock, Relativistic Heavy Ion Physics Vol. 23 (Springer, Berlin, 2010), p. 521.

[19] G. Aarts, G. F. Bonini, and C. Wetterich, On thermalization in classical scalar field theory, Nucl. Phys. B587, 403 (2000).

[20] S. Borsanyi, A. Patkos, and D. Sexty, Nonequilibrium Goldstone phenomenon in tachyonic preheating, Phys. Rev. D 68, 063512 (2003).

[21] C. Destri and H. J. de Vega, Ultraviolet cascade in the thermalization of the classical $\Phi^{4}$ theory in $3+1$ dimensions, Phys. Rev. D 73, 025014 (2006).

[22] D. Sexty and A. Patkos, Nonequilibrium Higgs transition in classical scalar electrodynamics, J. High Energy Phys. 10 (2005) 054.

[23] P. Romatschke and R. Venugopalan, The unstable glasma, Phys. Rev. D 74, 045011 (2006).

[24] J. Berges, S. Scheffler, S. Schlichting, and D. Sexty, Out of equilibrium dynamics of coherent non-Abelian gauge fields, Phys. Rev. D 85, 034507 (2012).

[25] F. Jin, T. Neuhaus, K. Michielsen, S. Miyashita, M. Novotny, M. I. Katsnelson, and H. De Raedt, Equilibration and thermalization of classical systems, New J. Phys. 15, 033009 (2013).

[26] J. Berges, B. Schenke, S. Schlichting, and R. Venugopalan, Turbulent thermalization process in high-energy heavy-ion collisions, Nucl. Phys. A931, 348 (2014).

[27] J. Berges, K. Boguslavski, S. Schlichting, and R. Venugopalan, Nonequilibrium fixed points in longitudinally expanding scalar theories: Infrared cascade, Bose condensation and a challenge for kinetic theory, Phys. Rev. D 92 , 096006 (2015).

[28] M. M. Homor and A. Jakovac, Shear viscosity of the $\Phi^{4}$ theory from classical simulation, Phys. Rev. D 92, 105011 (2015).

[29] C. R. Shalizi, Maximum likelihood estimation for q-exponential (Tsallis) distributions, arXiv:math/0701854v2.

[30] R. Sommer, A new way to set the energy scale in lattice gauge theories and its applications to the static force and alpha-s in SU(2) Yang-Mills theory, Nucl. Phys. B411, 839 (1994).

[31] A. Francis, O. Kaczmarek, M. Laine, T. Neuhaus, and H. Ohno, Critical point and scale setting in SU(3) plasma: An update, Phys. Rev. D 91, 096002 (2015).

[32] S. Necco and R. Sommer, The $N(f)=0$ heavy quark potential from short to intermediate distances, Nucl. Phys. B622, 328 (2002).

[33] M. Guagnelli, R. Sommer, and H. Wittig (ALPHA Collaboration), Precision computation of a low-energy reference scale in quenched lattice QCD, Nucl. Phys. B535, 389 (1998). 